\title{
CRITICAL PAIRS OF SEQUENCES OF A MIXED FRAME POTENTIAL
}

\author{
IVANA CARRIZO* AND SIGRID HEINEKEN ${ }^{\dagger}$
}

\begin{abstract}
The classical frame potential in a finite dimensional Hilbert space has been introduced by Benedetto and Fickus, who showed that all finite unit-norm tight frames can be characterized as the minimizers of this energy functional. This was the start point of a series of new results in frame theory, related to finding tight frames with determined length. The frame potential has been studied in the traditional setting as well as in the finite-dimensional fusion frame context. In this work we introduce the concept of mixed frame potential, which generalizes the notion of the Benedetto-Fickus frame potential. We study properties of this new potential, and give the structure of its critical pairs of sequences on a suitable restricted domain. For a given sequence $\left\{\alpha_{m}\right\}_{m=1, \ldots, N}$ in $K$, where $K$ is $\mathbb{R}$ or $\mathbb{C}$, we obtain necessary and sufficient conditions in order to have a dual pair of frames $\left\{f_{m}\right\}_{m=1, \ldots, N},\left\{g_{m}\right\}_{m=1, \ldots, N}$ such that $\left\langle f_{m}, g_{m}\right\rangle=\alpha_{m}$ for all $m=1, \ldots, N$.
\end{abstract}

Key words: Finite frames, frame potential, dual frames, Lagrange multipliers.

AMS subject classification: Primary: 42C15, 42C99, 42C40.

\section{INTRODUCTION}

Frames, which were introduced by Duffin and Schaeffer in [11, became essential for engineering and applied mathematics, specially for the purpose of signal processing and data transmission. Given a Hilbert space $\mathbb{H}$, a sequence $\left\{f_{m}\right\} \subset \mathbb{H}$ is a frame if there exist positive constants $A$ and $B$ that satisfy

$$
A\|f\|^{2} \leq \sum_{m}\left|\left\langle f, f_{m}\right\rangle\right|^{2} \leq B\|f\|^{2} \quad \forall f \in \mathbb{H} .
$$

If $A=B$ it is called a tight frame.

The main property of frames is that they provide reconstruction formulae where the coefficients are not necessarily unique, which is advantageous in situations that arise in signal processing [1. Particular frames such as wavelet and Gabor frames are described e.g. in [14, [9, [10, 6].

* NuHAG, Department of Mathematics, University of Vienna, Nordbergstrasse 15, A-1090 Vienna, Austria. E-mail: ivana.carrizo@univie.ac.at.

$\dagger$ Departamento de Matemática, Facultad de Ciencias Exactas y Naturales, Universidad de Buenos Aires, Pabellón I, Ciudad Universitaria, C1428EGA C.A.B.A., and IMAS, CONICET, Argentina. E-mail: sheinek@dm.uba.ar.

Correspondence to: Sigrid Heineken, Departamento de Matemática, Facultad de Ciencias Exactas y Naturales, Universidad de Buenos Aires, Pabellón I, Ciudad Universitaria, C1428EGA C.A.B.A., Argentina, tel/fax:+541145763335, E-mail: sheinek@dm.uba.ar, sigrid.heineken@gmail.com. 
Finite frames are used in many applications, where we often have to work in finite dimensional spaces, since they avoid the approximation problems that come up by truncating infinite frames. They have been studied for example in [2], 3], [12. In particular, finite tight frames are very useful to solve problems in Communication Theory, Information Theory, Sampling Theory, etc. [15, since the convergence of the provided decompositions is fast. The frame potential in $\mathbb{H}_{d}^{N}$ - where $\mathbb{H}_{d}$ is a finite dimensional Hilbert space - introduced in 2 by Benedetto and Fickus- turned out to be an important tool in frame theory. In our work we define a new concept of potential in $\mathbb{H}_{d}^{N} \times \mathbb{H}_{d}^{N}$. Whereas the Benedetto-Fickus potential measures the orthogonality of a system of vectors, our mixed frame potential quantifies in some sense the biorthogonality of two systems of vectors.

In $[3$ and 4 , the problem of finding tight frames with a prescribed norm is analyzed, which is related to the minimization of the Benedetto-Fickus frame potential. The Benedetto-Fickus frame potential has been also studied in the finite-dimensional fusion frame setting [5], 13 .

Given a sequence $\left\{\alpha_{m}\right\}_{m=1, \ldots, N}$, we study the mixed frame potential restricted to the pairs $\left(\left\{f_{m}\right\}_{m=1}^{N},\left\{g_{m}\right\}_{m=1}^{N}\right)$ such that $\left\langle f_{m}, g_{m}\right\rangle=\alpha_{m}$, and describe the critical pairs of sequences of this restricted potential. This turned out to be related to finding dual pairs of frames that satisfy $\left\langle f_{m}, g_{m}\right\rangle=\alpha_{m}$.

The paper is organized as follows. In the following section we give definitions and preliminaries that we will use later. In section 3 we present some properties of the mixed frame potential. Section 4 is devoted to characterize the structure of the critical pairs of sequences of the mixed frame potential, which leads to the result about necessary and sufficient conditions for the existence of dual frames with prescribed scalar products.

\section{Notation And Preliminaries}

Let $K$ be $\mathbb{R}$ or $\mathbb{C}$ and $\mathbb{H}_{d}$ a $d$-dimensional Hilbert space over $K$. Let $\left\{f_{m}\right\}_{m=1}^{N}$ and $\left\{g_{m}\right\}_{m=1}^{N}$ be sequences in $\mathbb{H}_{d}$. The synthesis operator for $\left\{f_{m}\right\}_{m=1}^{N}$ is given by

$$
T: K^{N} \rightarrow \mathbb{H}_{d}, T\left(\left\{c_{m}\right\}_{m=1}^{N}\right)=\sum_{m=1}^{N} c_{m} f_{m}
$$

and the analysis operator for $\left\{f_{m}\right\}_{m=1}^{N}$ by

$$
T^{\star}: \mathbb{H}_{d} \rightarrow K^{N}, T^{\star}(f)=\left\{\left\langle f, f_{m}\right\rangle\right\}_{m=1}^{N} .
$$

We will denote with $U$ and $U^{\star}$ the synthesis and respectively analysis operator of $\left\{g_{m}\right\}_{m=1}^{N}$. We denominate $T U^{\star}$ and $U T^{\star}$ the mixed frame operators:

For $f \in \mathbb{H}_{d}$ we have

$$
T U^{\star}(f)=\sum_{m=1}^{N}\left\langle f, g_{m}\right\rangle f_{m}, \text { and } U T^{\star}(f)=\sum_{m=1}^{N}\left\langle f, f_{m}\right\rangle g_{m} .
$$


Two sequences $\left\{f_{m}\right\}_{m=1}^{N}$ and $\left\{g_{m}\right\}_{m=1}^{N}$ are dual frames if

$$
f=\sum_{m=1}^{N}\left\langle f, g_{m}\right\rangle f_{m} \forall f \in \mathbb{H}_{d} \text { or } f=\sum_{m=1}^{N}\left\langle f, f_{m}\right\rangle g_{m} \forall f \in \mathbb{H}_{d}
$$

In terms of the operators $T$ and $U$, (2) means that $T U^{\star}=I$ or $U T^{\star}=I$.

Definition 1. Let $\widetilde{F P}: \mathbb{H}_{d}^{N} \times \mathbb{H}_{d}^{N} \longrightarrow K$,

$$
\widetilde{F P}\left(\left\{f_{m}\right\}_{m=1}^{N},\left\{g_{m}\right\}_{m=1}^{N}\right)=\sum_{m=1}^{N} \sum_{n=1}^{N}\left\langle f_{m}, g_{n}\right\rangle\left\langle f_{n}, g_{m}\right\rangle .
$$

We call $\widetilde{F P}$ the mixed frame potential of $\left(\left\{f_{m}\right\}_{m=1}^{N},\left\{g_{m}\right\}_{m=1}^{N}\right) \in \mathbb{H}_{d}^{N} \times \mathbb{H}_{d}^{N}$.

Observe that for the case that $\left\{f_{m}\right\}_{m=1}^{N}=\left\{g_{m}\right\}_{m=1}^{N}$, the mixed frame potential is equal to

$$
F P\left(\left\{f_{m}\right\}_{m=1}^{N}\right)=\sum_{m=1}^{N} \sum_{n=1}^{N}\left|\left\langle f_{m}, f_{n}\right\rangle\right|^{2}
$$

which is the traditional Benedetto-Fickus frame potential of $\left\{f_{m}\right\}_{m=1}^{N}$.

Given a sequence $\left\{\alpha_{m}\right\}_{m=1}^{N} \subset K$ we define

$\tilde{S}\left(\left\{\alpha_{m}\right\}_{m=1}^{N}\right)=\left\{\left(\left\{f_{m}\right\}_{m=1}^{N},\left\{g_{m}\right\}_{m=1}^{N}\right) \in \mathbb{H}_{d}^{N} \times \mathbb{H}_{d}^{N}:\left\langle f_{m}, g_{m}\right\rangle=\alpha_{m} \forall m=1, \ldots, N\right\}$

\section{Mixed Frame Potential}

We will see next that the mixed frame potential can also be written as the trace of the square of the corresponding mixed frame operator, i.e. it is the square of the Hilbert-Schmidt norm of the mixed frame operator.

Lemma 1. For any pair $\left(\left\{f_{m}\right\}_{m=1}^{N},\left\{g_{m}\right\}_{m=1}^{N}\right) \in \mathbb{H}_{d}^{N} \times \mathbb{H}_{d}^{N}$ with corresponding mixed frame operator $T U^{\star}$,

$$
\widetilde{F P}\left(\left\{f_{m}\right\}_{m=1}^{N},\left\{g_{m}\right\}_{m=1}^{N}\right)=\operatorname{Tr}\left(\left(T U^{\star}\right)^{2}\right)=\sum_{n=1}^{d} \lambda_{n}^{2}
$$

where $\left\{\lambda_{n}\right\}_{n=1}^{d}$ are the eigenvalues of $T U^{\star}$. 
Proof. Let $\left\{e_{n}\right\}_{n=1}^{d}$ be an orthonormal basis of $\mathbb{H}_{d}$.

$$
\begin{aligned}
\widetilde{F P}\left(\left\{f_{m}\right\}_{m=1}^{N},\left\{g_{m}\right\}_{m=1}^{N}\right) & =\sum_{m=1}^{N} \sum_{n=1}^{N}\left\langle f_{m}, g_{n}\right\rangle\left\langle f_{n}, g_{m}\right\rangle \\
& =\sum_{m=1}^{N} \sum_{n=1}^{N}\left\langle\sum_{l=1}^{d}\left\langle f_{m}, e_{l}\right\rangle e_{l}, g_{n}\right\rangle\left\langle f_{n}, g_{m}\right\rangle \\
& =\sum_{m=1}^{N} \sum_{n=1}^{N} \sum_{l=1}^{d}\left\langle f_{m}, e_{l}\right\rangle\left\langle e_{l}, g_{n}\right\rangle\left\langle f_{n}, g_{m}\right\rangle \\
& =\sum_{l=1}^{d}\left\langle\sum_{n=1}^{N}\left\langle e_{l}, g_{n}\right\rangle f_{n}, \sum_{m=1}^{N}\left\langle e_{l}, f_{m}\right\rangle g_{m}\right\rangle \\
& =\sum_{l=1}^{d}\left\langle T U^{\star} e_{l}, U T^{\star} e_{l}\right\rangle=\sum_{l=1}^{d}\left\langle\left(U T^{\star}\right)^{\star} T U^{\star} e_{l}, e_{l}\right\rangle \\
& =\sum_{l=1}^{d}\left\langle\left(T U^{\star}\right)^{2} e_{l}, e_{l}\right\rangle=\operatorname{Tr}\left(\left(T U^{\star}\right)^{2}\right)
\end{aligned}
$$

Let $\left\{\lambda_{n}\right\}_{n=1}^{d}$ denote the eigenvalues of $T U^{\star}$, counting multiplicities. Since the eigenvalues of $\left(T U^{\star}\right)^{2}$ are $\left\{\lambda_{n}^{2}\right\}_{n=1}^{d}$ we have that

$$
\operatorname{Tr}\left(\left(T U^{\star}\right)^{2}\right)=\sum_{n=1}^{d} \lambda_{n}^{2} .
$$

Remark 1. Observe that

$$
\widetilde{F P}\left(\left\{g_{m}\right\}_{m=1}^{N},\left\{f_{m}\right\}_{m=1}^{N}\right)=\operatorname{Tr}\left(\left(U^{\star} T\right)^{2}\right)=\sum_{n=1}^{d}{\overline{\lambda_{n}}}^{2} .
$$

Note that the previous result allows to compute the mixed frame potential very easily for example for a pair $\left(\left\{f_{m}\right\}_{m=1}^{N},\left\{g_{m}\right\}_{m=1}^{N}\right)$ such that $T U^{*}=A$ Id with $A \in K$. In this case, $\widetilde{F P}\left(\left\{f_{m}\right\}_{m=1}^{N},\left\{g_{m}\right\}_{m=1}^{N}\right)=A^{2} d$.

Also, the previous representation of the mixed frame potential allows us to study in more detail some of its properties, as we will see in the following proposition.

Proposition 1. Let $\left\{\alpha_{m}\right\}_{m=1}^{N} \subset K$ and $\left(\left\{f_{m}\right\}_{m=1}^{N},\left\{g_{m}\right\}_{m=1}^{N}\right) \in \tilde{S}\left(\left\{\alpha_{m}\right\}_{m=1}^{N}\right)$.

(1) If all the eigenvalues of $T U^{\star}$ are real, then $\widetilde{F P}\left(\left\{f_{m}\right\}_{m=1}^{N},\left\{g_{m}\right\}_{m=1}^{N}\right)$ and $\sum_{m=1}^{N} \alpha_{m}$ are real and

$$
\widetilde{F P}\left(\left\{f_{m}\right\}_{m=1}^{N},\left\{g_{m}\right\}_{m=1}^{N}\right) \geq \frac{1}{d}\left(\sum_{m=1}^{N} \alpha_{m}\right)^{2} .
$$


(2) If all the eigenvalues of $T U^{\star}$ are imaginary, then $\widetilde{F P}\left(\left\{f_{m}\right\}_{m=1}^{N},\left\{g_{m}\right\}_{m=1}^{N}\right)$ is real and $\sum_{m=1}^{N} \alpha_{m}$ is imaginary and

$$
\widetilde{F P}\left(\left\{f_{m}\right\}_{m=1}^{N},\left\{g_{m}\right\}_{m=1}^{N}\right) \leq \frac{1}{d}\left(\sum_{m=1}^{N} \alpha_{m}\right)^{2}
$$

(3) If $T U^{*}$ has only one eigenvalue, then

$$
\widetilde{F P}\left(\left\{f_{m}\right\}_{m=1}^{N},\left\{g_{m}\right\}_{m=1}^{N}\right)=\frac{1}{d}\left(\sum_{m=1}^{N} \alpha_{m}\right)^{2} .
$$

In particular, this happens if $T U^{*}=\frac{1}{d}\left(\sum_{m=1}^{N} \alpha_{m}\right) \mathrm{Id}$.

Proof. By the preceding lemma we know that if $\left(\left\{f_{m}\right\}_{m=1}^{N},\left\{g_{m}\right\}_{m=1}^{N}\right) \in \mathbb{H}_{d}^{N} \times \mathbb{H}_{d}^{N}$,

$$
\begin{aligned}
\widetilde{F P}\left(\left\{f_{m}\right\}_{m=1}^{N},\left\{g_{m}\right\}_{m=1}^{N}\right) & =\sum_{n=1}^{d} \lambda_{n}^{2} \\
& =\sum_{n=1}^{d}\left(\left(\operatorname{Re}\left(\lambda_{n}\right)\right)^{2}-\left(\operatorname{Im}\left(\lambda_{n}\right)\right)^{2}\right)+2 i \sum_{n=1}^{d} \operatorname{Re}\left(\lambda_{n}\right) \operatorname{Im}\left(\lambda_{n}\right)
\end{aligned}
$$

where $\left\{\lambda_{n}\right\}_{n=1}^{d}$ are the eigenvalues of $T U^{\star}$.

Let $\left\{e_{n}\right\}_{n=1}^{d}$ be an orthonormal basis for $\mathbb{H}_{d}$. If $\left(\left\{f_{m}\right\}_{m=1}^{N},\left\{g_{m}\right\}_{m=1}^{N}\right) \in \tilde{S}\left(\left\{\alpha_{m}\right\}_{m=1}^{N}\right)$ the trace of the mixed frame operator satisfies

$$
\begin{aligned}
\sum_{n=1}^{d} \lambda_{n} & =\operatorname{Tr}\left(T U^{\star}\right)=\sum_{n=1}^{d}\left\langle T U^{\star} e_{n}, e_{n}\right\rangle=\sum_{n=1}^{d}\left\langle\sum_{m=1}^{N}\left\langle e_{n}, g_{m}\right\rangle f_{m}, e_{n}\right\rangle \\
& =\sum_{n=1}^{d} \sum_{m=1}^{N}\left\langle e_{n}, g_{m}\right\rangle\left\langle f_{m}, e_{n}\right\rangle=\sum_{m=1}^{N} \sum_{n=1}^{d}\left\langle e_{n}, g_{m}\right\rangle\left\langle f_{m}, e_{n}\right\rangle \\
& =\sum_{m=1}^{N}\left\langle f_{m}, g_{m}\right\rangle=\sum_{m=1}^{N} \alpha_{m} .
\end{aligned}
$$

So, in order to study possible extrema for the real or the imaginary part of $\widetilde{F P}$ : $\tilde{S}\left(\left\{\alpha_{m}\right\}_{m=1}^{N}\right) \longrightarrow K$, we will first consider the critical points of the functions

$$
\mathcal{R}\left(\lambda_{1}, \ldots, \lambda_{d}\right)=\mathcal{R}\left(\operatorname{Re}\left(\lambda_{1}\right), \ldots, \operatorname{Re}\left(\lambda_{d}\right), \operatorname{Im}\left(\lambda_{1}\right), \ldots, \operatorname{Im}\left(\lambda_{d}\right)\right)=\sum_{n=1}^{d}\left(\operatorname{Re}\left(\lambda_{n}\right)\right)^{2}-\left(\operatorname{Im}\left(\lambda_{n}\right)\right)^{2}
$$

and

$$
\mathcal{I}\left(\lambda_{1}, \ldots, \lambda_{d}\right)=\mathcal{I}\left(\operatorname{Re}\left(\lambda_{1}\right), \ldots, \operatorname{Re}\left(\lambda_{d}\right), \operatorname{Im}\left(\lambda_{1}\right), \ldots, \operatorname{Im}\left(\lambda_{d}\right)\right)=2 \sum_{n=1}^{d} \operatorname{Re}\left(\lambda_{n}\right) \operatorname{Im}\left(\lambda_{n}\right)
$$

restricted to the set $\Lambda \subset \mathbb{C}^{d} \simeq \mathbb{R}^{2 d}$, where $\left(\lambda_{1}, \ldots, \lambda_{d}\right) \in \Lambda$ if and only if

$$
\sum_{n=1}^{d} \operatorname{Re}\left(\lambda_{n}\right)=\operatorname{Re}\left(\sum_{m=1}^{N} \alpha_{m}\right) \text { and } \sum_{n=1}^{d} \operatorname{Im}\left(\lambda_{n}\right)=\operatorname{Im}\left(\sum_{m=1}^{N} \alpha_{m}\right) .
$$


Using Lagrange multipliers for this constrained problem, we obtain that if $\left(\lambda_{1}, \ldots, \lambda_{d}\right)$ is a critical point of $\mathcal{R}$ or $\mathcal{I}$ restricted to $\Lambda$, then

$$
\lambda_{1}=\lambda_{2}=\ldots=\lambda_{d}=\frac{1}{d} \sum_{m=1}^{N} \alpha_{m} .
$$

Furthermore, in this case it can be seen that

(i) if $\operatorname{Im}\left(\lambda_{1}, \ldots, \lambda_{d}\right)=0$ then $\left(\lambda_{1}, \ldots, \lambda_{d}\right)$ is a minimum of $\mathcal{R}$ restricted to $\Lambda$ and $\mathcal{I}\left(\lambda_{1}, \ldots, \lambda_{d}\right)=0$

(ii) if $\operatorname{Re}\left(\lambda_{1}, \ldots, \lambda_{d}\right)=0$ then $\left(\lambda_{1}, \ldots, \lambda_{d}\right)$ is a maximum of $\mathcal{R}$ restricted to $\Lambda$ and $\mathcal{I}\left(\lambda_{1}, \ldots, \lambda_{d}\right)=0$

(iii) if $\operatorname{Re}\left(\lambda_{1}, \ldots, \lambda_{d}\right) \neq 0$ and $\operatorname{Im}\left(\lambda_{1}, \ldots, \lambda_{d}\right) \neq 0$, then $\left(\lambda_{1}, \ldots, \lambda_{d}\right)$ is a saddle point of $\mathcal{R}$ as well as of $\mathcal{I}$ restricted to $\Lambda$.

Thus, for any $\left(\left\{f_{m}\right\}_{m=1}^{N},\left\{g_{m}\right\}_{m=1}^{N}\right) \in \tilde{S}\left(\left\{\alpha_{m}\right\}_{m=1}^{N}\right)$ such that all the eigenvalues of $T U^{\star}$ are real, we have that $\overline{F P}\left(\left\{f_{m}\right\}_{m=1}^{N},\left\{g_{m}\right\}_{m=1}^{N}\right)$ and $\sum_{m=1}^{N} \alpha_{m}$ are real and

$$
\left.\widetilde{F P}\left(\left\{f_{m}\right\}_{m=1}^{N},\left\{g_{m}\right\}_{m=1}^{N}\right)=\sum_{n=1}^{d} \lambda_{n}^{2} \geq \frac{1}{d}\left(\sum_{m=1}^{N} \alpha_{m}\right)\right)^{2},
$$

and for any $\left(\left\{f_{m}\right\}_{m=1}^{N},\left\{g_{m}\right\}_{m=1}^{N}\right) \in \tilde{S}\left(\left\{\alpha_{m}\right\}_{m=1}^{N}\right)$ such that all the eigenvalues of $T U^{\star}$ are imaginary, $\widetilde{F P}\left(\left\{f_{m}\right\}_{m=1}^{N},\left\{g_{m}\right\}_{m=1}^{N}\right)$ is real and $\sum_{m=1}^{N} \alpha_{m}$ is imaginary and

$$
\operatorname{Im}\left(\widetilde{F P}\left(\left\{f_{m}\right\}_{m=1}^{N},\left\{g_{m}\right\}_{m=1}^{N}\right)\right)=\sum_{n=1}^{d} \lambda_{n}^{2} \leq \frac{1}{d}\left(\sum_{m=1}^{N} \alpha_{m}\right)^{2}
$$

If $T U^{\star}$ has only one eigenvalue $\lambda$, then $\lambda=\frac{1}{d}\left(\sum_{m=1}^{N} \alpha_{m}\right)$ since $\lambda \in \Lambda$, and so $\widetilde{F P}\left(\left\{f_{m}\right\}_{m=1}^{N},\left\{g_{m}\right\}_{m=1}^{N}\right)=\sum_{n=1}^{d} \lambda_{n}^{2}=\frac{1}{d}\left(\sum_{m=1}^{N} \alpha_{m}\right)^{2}$.

Remark 2. Note that the bounds in (1) and (2) of Proposition 1 are not necessarily achieved, but are attained when $T U^{*}$ has only one eigenvalue.

Our next step is to study critical pairs of sequences of our mixed frame potential.

\section{CRitical pairs of Sequences of the mixed frame potential}

We show now that if the mixed frame operator is the identity operator times a constant, then the sequence $\left\{\alpha_{m}\right\}_{m=1, \ldots, N}$ satisfies an equality.

Proposition 2. Let $\left(\left\{f_{m}\right\}_{m=1}^{N},\left\{g_{m}\right\}_{m=1}^{N}\right) \in \tilde{S}\left(\left\{\alpha_{m}\right\}_{m=1}^{N}\right.$ be such that $T U^{\star}=A \mathrm{Id}$ with $A \in K$. Then $\frac{1}{d} \sum_{i=1}^{N} \alpha_{i}=A$. 
Proof. Let $\left\{e_{n}\right\}_{n=1}^{d}$ be an orthonormal basis in $\mathbb{H}_{d}$. Since $A \mathrm{Id}=T U^{\star}$ we have that

$$
\begin{aligned}
\frac{1}{d} \sum_{m=1}^{N} \alpha_{m} & =\frac{1}{d} \sum_{m=1}^{N}\left\langle f_{m}, g_{m}\right\rangle=\frac{1}{d} \sum_{m=1}^{N}\left\langle\sum_{j=1}^{d}\left\langle f_{m}, e_{j}\right\rangle e_{j}, g_{m}\right\rangle \\
& =\frac{1}{d} \sum_{m=1}^{N} \sum_{j=1}^{d}\left\langle f_{m}, e_{j}\right\rangle\left\langle e_{j}, g_{m}\right\rangle=\frac{1}{d} \sum_{j=1}^{d} A\left\langle e_{j}, e_{j}\right\rangle=A
\end{aligned}
$$

In order to state the following results we will need some definitions.

Let $\mathcal{L}$ be a finite index set.

Definition 2. We call $\left\{f_{m}\right\}_{m \in \mathcal{L}} \subset \mathbb{H}_{d}$ and $\left\{g_{m}\right\}_{m \in \mathcal{L}} \subset \mathbb{H}_{d}$ generalized biorthogonal sequences if there exists $\left\{\alpha_{m}\right\}_{m \in \mathcal{L}} \subset K_{\neq 0}$ such that

$$
\begin{cases}\left\langle f_{n}, g_{m}\right\rangle=0, & \text { for all } n \neq m \\ \left\langle f_{m}, g_{m}\right\rangle=\alpha_{m}, & \text { for all } m \in \mathcal{L} .\end{cases}
$$

Definition 3. Let $A \in K$. We say $\left\{f_{m}\right\}_{m \in \mathcal{L}} \subset \mathbb{H}_{d}$ and $\left\{g_{m}\right\}_{m \in \mathcal{L}} \subset \mathbb{H}_{d}$ are $A$ generalized dual frames if

$$
\begin{cases}\sum_{m \in \mathcal{L}}\left\langle f, g_{m}\right\rangle f_{m}=A f, & \text { for all } f \in \operatorname{span}\left\{f_{m}\right\}_{m \in \mathcal{L}} \text { and } \\ \sum_{m \in \mathcal{L}}\left\langle f, f_{m}\right\rangle g_{m}=\bar{A} f, & \text { for all } f \in \operatorname{span}\left\{g_{m}\right\}_{m \in \mathcal{L}}\end{cases}
$$

In the following we will see that the critical points of the real or the imaginary part of the restricted mixed frame potential satisfy certain Lagrange equations.

Proposition 3. Let $\left\{\alpha_{n}\right\}_{m=1}^{N} \subset K$. If $\left(\left\{f_{m}\right\}_{m=1}^{N},\left\{g_{m}\right\}_{m=1}^{N}\right)$ is a local extrema or a saddle point of the real or the imaginary part of the mixed frame potential $\widetilde{F P}: \tilde{S}\left(\left\{\alpha_{m}\right\}_{m=1}^{N}\right) \longrightarrow K$, then for each $m=1, \ldots, N$ there exists $c \in K$ such that

$$
\sum_{n=1, n \neq m}^{N}\left\langle f_{m}, g_{n}\right\rangle f_{n}=c f_{m} \text { and } \sum_{n=1, n \neq m}^{N}\left\langle g_{m}, f_{n}\right\rangle g_{n}=\bar{c} g_{m}
$$

Proof. Consider the $m$-th mixed frame potential denoted by $\widetilde{F P}_{m}$, where

$$
\widetilde{F P}_{m}(f, g)=\left\langle f_{m}, g_{m}\right\rangle^{2}+\sum_{n \neq m}\left\langle f_{n}, g\right\rangle\left\langle f, g_{n}\right\rangle+\widetilde{F P}\left(\left\{f_{n}\right\}_{n \neq m},\left\{g_{n}\right\}_{n \neq m}\right) .
$$

Since $\left(\left\{f_{m}\right\}_{m=1}^{N},\left\{g_{m}\right\}_{m=1}^{N}\right)$ is a local extrema or a saddle point of the real or the imaginary part of the frame potential $\widetilde{F P}$ restricted to $\tilde{S}\left(\left\{\alpha_{m}\right\}_{m=1}^{N}\right)$, we have that $\left(f_{m}, g_{m}\right)$ is a local extrema or a saddle point of the real or the imaginary part of $\widetilde{F P}_{m}$ in $S\left(\alpha_{m}\right)=\left\{(f, g) \in \mathcal{H} \times \mathcal{H}:\langle f, g\rangle=\alpha_{m}\right\}$, where

$$
\widetilde{F P}_{m}(f, g)=\alpha_{m}^{2}+\sum_{n \neq m}\left\langle f_{n}, g\right\rangle\left\langle f, g_{n}\right\rangle+\sum_{n=1, n \neq m}^{N} \sum_{r=1, r \neq m}^{N}\left\langle f_{n}, g_{r}\right\rangle\left\langle g_{r}, f_{n}\right\rangle \text {. }
$$


Hence, the corresponding several variable constrained problem must be solved. Using Lagrange multipliers, it can be seen that there exist $c_{1}, c_{2} \in \mathbb{R}$ such that

$$
\left.(I) \nabla \operatorname{Re}\left(\widetilde{F P}{ }_{m}\right)(f, g)\right|_{\left(f_{m}, g_{m}\right)}=\left.c_{1} \nabla \operatorname{Re}(\langle f, g\rangle)\right|_{\left(f_{m}, g_{m}\right)}+\left.c_{2} \nabla \operatorname{Im}(\langle f, g\rangle)\right|_{\left(f_{m}, g_{m}\right)}
$$

or there exist $c_{2}, c_{3} \in R$ such that

$$
\left.(I I) \nabla \operatorname{Im}\left(\widetilde{F P}_{m}\right)(f, g)\right|_{\left(f_{m}, g_{m}\right)}=\left.c_{3} \nabla \operatorname{Re}(\langle f, g\rangle)\right|_{\left(f_{m}, g_{m}\right)}+\left.c_{4} \nabla \operatorname{Im}(\langle f, g\rangle)\right|_{\left(f_{m}, g_{m}\right)} .
$$

From $(I)$ we have the following equations

(i) $\left.\nabla_{\operatorname{Re}(f)} \operatorname{Re}\left(\widetilde{F P_{m}}\right)(f, g)\right|_{\left(f_{m}, g_{m}\right)}=\left.c_{1} \nabla_{\operatorname{Re}(f)} \operatorname{Re}(\langle f, g\rangle)\right|_{\left(f_{m}, g_{m}\right)}+\left.c_{2} \nabla_{R e(f)} \operatorname{Im}(\langle f, g\rangle)\right|_{\left(f_{m}, g_{m}\right)}$,

(ii) $\left.\nabla_{I m(f)} \operatorname{Re}(\widetilde{F P} m)(f, g)\right|_{\left(f_{m}, g_{m}\right)}=\left.c_{1} \nabla_{I m(f)} \operatorname{Re}(\langle f, g\rangle)\right|_{\left(f_{m}, g_{m}\right)}+\left.c_{2} \nabla_{I m(f)} \operatorname{Im}(\langle f, g\rangle)\right|_{\left(f_{m}, g_{m}\right)}$,

(iii) $\left.\nabla_{\operatorname{Re}(g)} \operatorname{Re}\left(\widetilde{F P_{m}}\right)(f, g)\right|_{\left(f_{m}, g_{m}\right)}=\left.c_{1} \nabla_{\operatorname{Re}(g)} \operatorname{Re}(\langle f, g\rangle)\right|_{\left(f_{m}, g_{m}\right)}+\left.c_{2} \nabla_{\operatorname{Re}(g)} \operatorname{Im}(\langle f, g\rangle)\right|_{\left(f_{m}, g_{m}\right)}$,

(iv) $\left.\nabla_{I m(g)} \operatorname{Re}\left(\widetilde{F P}_{m}\right)(f, g)\right|_{\left(f_{m}, g_{m}\right)}=\left.c_{1} \nabla_{\operatorname{Im}(g)} \operatorname{Re}(\langle f, g\rangle)\right|_{\left(f_{m}, g_{m}\right)}+\left.c_{2} \nabla_{\operatorname{Im}(g)} \operatorname{Im}(\langle f, g\rangle)\right|_{\left(f_{m}, g_{m}\right)}$,

Hence, from (i) and (ii)

$$
\begin{aligned}
& \operatorname{Re}\left(\sum_{n=1, n \neq m}^{N}\left\langle g_{m}, f_{n}\right\rangle g_{n}\right)=c_{1} \operatorname{Re}\left(g_{m}\right)-c_{2} \operatorname{Im}\left(g_{m}\right), \\
& \operatorname{Im}\left(\sum_{n=1, n \neq m}^{N}\left\langle g_{m}, f_{n}\right\rangle g_{n}\right)=c_{1} \operatorname{Im}\left(g_{m}\right)+c_{2} \operatorname{Re}\left(g_{m}\right)
\end{aligned}
$$

and from (iii) and (iv)

$$
\begin{aligned}
& \operatorname{Re}\left(\sum_{n=1, n \neq m}^{N}\left\langle f_{m}, g_{n}\right\rangle f_{n}\right)=c_{1} \operatorname{Re}\left(f_{m}\right)+c_{2} \operatorname{Im}\left(f_{m}\right), \\
& \operatorname{Im}\left(\sum_{n=1, n \neq m}^{N}\left\langle f_{m}, g_{n}\right\rangle f_{n}\right)=c_{1} \operatorname{Im}\left(f_{m}\right)-c_{2} \operatorname{Re}\left(f_{m}\right),
\end{aligned}
$$

which yields,

$$
\sum_{n=1, n \neq m}^{N}\left\langle g_{m}, f_{n}\right\rangle g_{n}=c_{1} g_{m}+i c_{2} g_{m}=\left(c_{1}+i c_{2}\right) g_{m}
$$

and

$$
\sum_{n=1, n \neq m}^{N}\left\langle f_{m}, g_{n}\right\rangle f_{n}=c_{1} f_{m}-i c_{2} f_{m}=\left(c_{1}-i c_{2}\right) f_{m},
$$

so we obtain the desired result if we take $c=c_{1}+i c_{2}$.

Observe that in a similar way we can obtain from $(I I)$ that

$$
\sum_{n=1, n \neq m}^{N}\left\langle g_{m}, f_{n}\right\rangle g_{n}=\left(c_{4}-i c_{3}\right) g_{m}
$$


and

$$
\sum_{n=1, n \neq m}^{N}\left\langle f_{m}, g_{n}\right\rangle f_{n}=\left(c_{4}+i c_{3}\right) f_{m},
$$

which implies in particular that if $\left(\left\{f_{m}\right\}_{m=1}^{N},\left\{g_{m}\right\}_{m=1}^{N}\right)$ is a local extrema or a saddle point of the real and the imaginary part of the restricted mixed frame potential, then $c_{4}=c_{1}$ and $c_{3}=-c_{2}$.

Definition 4. Let $\left\{\alpha_{m}\right\}_{m=1}^{N} \subset K$. We say that $\left(\left\{f_{n}\right\}_{m=1}^{N},\left\{g_{n}\right\}_{m=1}^{N}\right) \in \tilde{S}\left(\left\{\alpha_{m}\right\}_{m=1}^{N}\right)$ is a critical pair of sequences if for each $m=1, \ldots, N$ there exists $c \in K$ such that (77) is satisfied.

Now we are ready to provide a structure of these critical pairs of sequences:

Theorem 1. Let $\left\{\alpha_{m}\right\}_{m=1}^{N} \subset K$. If $\left(\left\{f_{m}\right\}_{m=1}^{N},\left\{g_{m}\right\}_{m=1}^{N}\right)$ is a critical pair of sequences, then

(1) for each $m \in\{1, \ldots, N\}, f_{m}$ is an eigenvector of $T U^{\star}$ and $g_{m}$ is an eigenvector of $U T^{\star}$, and the corresponding eigenvalues are conjugates.

(2) for $\left\{\lambda_{j}\right\}_{j=1}^{J}$ the sequence of distinct eigenvalues of $T U^{\star}$, there exists a sequence of indexing sets $\left\{I_{j}\right\}_{j=1}^{J}$ with $\bigcup_{j=1}^{J} I_{j}=\{1, \ldots, N\}$, such that $\left\{f_{m}\right\}_{m \in I_{j}}$ and $\left\{g_{m}\right\}_{m \in I_{j}}$ are $\lambda_{j}$-generalized dual frames.

Proof. (1) Since $\left(\left\{f_{n}\right\}_{m=1}^{N},\left\{g_{n}\right\}_{m=1}^{N}\right) \in \tilde{S}\left(\left\{\alpha_{m}\right\}_{m=1}^{N}\right)$ is a critical pair of sequences, for $m \in 1, \ldots, N$ there exists $c \in K$ such that

$$
\sum_{n=1, n \neq m}^{N}\left\langle f_{m}, g_{n}\right\rangle f_{n}=c f_{m} \text { and } \sum_{n=1, n \neq m}^{N}\left\langle g_{m}, f_{n}\right\rangle g_{n}=\bar{c} g_{m} .
$$

So,

$$
T U^{\star} f_{m}=\left\langle f_{m}, g_{m}\right\rangle f_{m}+\sum_{n=1, n \neq m}^{N}\left\langle f_{m}, g_{n}\right\rangle f_{n}=\alpha_{m} f_{m}+c f_{m}=\left(\alpha_{m}+c\right) f_{m},
$$

and

$$
U T^{*} g_{m}=\sum_{n=1, n \neq m}^{N}\left\langle g_{m}, f_{n}\right\rangle g_{n}=\left\langle g_{m}, f_{m}\right\rangle g_{m}+\sum_{n=1, n \neq m}^{N}\left\langle g_{m}, f_{n}\right\rangle g_{n}=\left(\overline{\alpha_{m}}+\bar{c}\right) g_{m},
$$

i.e. $f_{m}$ is an eigenvector of $T U^{*}$ and $g_{m}$ is an eigenvector of $U T^{*}$ and the eigenvalues are conjugates.

(2) Let $\left\{\lambda_{j}\right\}_{j=1}^{J}$ be the sequence of distinct eigenvalues of $T U^{\star}$. Since $\left(T U^{\star}\right)^{\star}=$ $U T^{\star}$, the eigenvalues of $U T^{\star}$ are the conjugates of the eigenvalues of $T U^{\star}$. We call $\left\{R_{j}\right\}_{j=1}^{J}$ the set of all right eigenvectors of $T U^{\star}$, and $\left\{L_{j}\right\}_{j=1}^{J}$ the set of all left eigenvectors of $T U^{\star}$, i.e. for each $j=1, \ldots, J$ we have:

$$
\begin{aligned}
R_{j} & =\left\{f \in \mathbb{H}_{d}: T U^{\star} f=\lambda_{j} f\right\}=\left\{f \in \mathbb{H}_{d}: f^{\star} U T^{\star}=\overline{\lambda_{j}} f^{\star}\right\} \\
L_{j} & =\left\{g \in \mathbb{H}_{d}: g^{\star} T U^{\star}=\lambda_{j} g^{\star}\right\}=\left\{g \in \mathbb{H}_{d}: U T^{\star} g=\overline{\lambda_{j}} g\right\}
\end{aligned}
$$

We know that if $i \neq j$ then $R_{i} \perp L_{j}$. 
Let $\left\{I_{j}\right\}_{j=1}^{J}$ be the sequence of indexing sets given by

$$
I_{j}=\left\{m \in\{1, \ldots, N\}: T U^{\star} f_{m}=\lambda_{j} f_{m} \text { and } U T^{\star} g_{m}=\overline{\lambda_{j}} g_{m}\right\} .
$$

Take $j \in\{1, \ldots, J\}$ and $f \in R_{j}$. If $m \notin I_{j}$ then $m \in I_{i}$ for some $i \neq j$, hence $g_{m} \in L_{i}$ following that $\left\langle f, g_{m}\right\rangle=0$. This yields

$$
\sum_{m \in I_{j}}\left\langle f, g_{m}\right\rangle f_{m}=T U^{*} f=\lambda_{j} f
$$

Analogously we obtain that for $f \in L_{j}$

$$
\sum_{m \in I_{j}}\left\langle f, f_{m}\right\rangle g_{m}=U T^{*} f=\overline{\lambda_{j}} f,
$$

So, since $\operatorname{span}\left\{f_{m}\right\}_{m \in I_{j}} \subseteq R_{j}$, and $\operatorname{span}\left\{g_{m}\right\}_{m \in I_{j}} \subseteq L_{j}$, we have that

$$
\left\{\begin{array}{l}
\sum_{m \in I_{j}}\left\langle f, g_{m}\right\rangle f_{m}=T U^{*} f=\lambda_{j} f, \quad \text { for all } f \in \operatorname{span}\left\{f_{m}\right\}_{m \in I_{j}} \text { and } \\
\sum_{m \in I_{j}}\left\langle f, f_{m}\right\rangle g_{m}=U T^{*} f=\overline{\lambda_{j}} f, \quad \text { for all } f \in \operatorname{span}\left\{g_{m}\right\}_{m \in I_{j}},
\end{array}\right.
$$

i.e. $\left\{f_{m}\right\}_{m \in I_{j}}$ and $\left\{g_{m}\right\}_{m \in I_{j}}$ are $\lambda_{j}$-generalized dual frames. Moreover, we proved that if $\lambda_{j} \neq 0$ then $\operatorname{span}\left\{f_{m}\right\}_{m \in I_{j}}=R_{j}$ and $\operatorname{span}\left\{g_{m}\right\}_{m \in I_{j}}=L_{j}$.

Now we describe the structure of the pairs that are local extrema of the real and the imaginary part of the restricted frame potential. As we will see in Proposition 4 under certain conditions the same structure is also valid for pairs that are local extrema of the real or the imaginary part of the restricted frame potential.

Theorem 2. Let $\left\{\alpha_{n}\right\}_{n=1}^{N} \subset K_{\neq 0}$. Then every pair $\left(\left\{f_{m}\right\}_{m=1}^{N},\left\{g_{m}\right\}_{m=1}^{N}\right)$ which is a local extrema of the real and the imaginary part of the mixed frame potential $\widetilde{F P}: \tilde{S}\left(\left\{\alpha_{m}\right\}_{m=1}^{N}\right) \longrightarrow K$, can be decomposed as

$$
\left(\left\{f_{m}\right\}_{m \in \mathcal{I}^{c}} \cup\left\{f_{m}\right\}_{m \in \mathcal{I}},\left\{g_{m}\right\}_{m \in \mathcal{I}^{c}} \cup\left\{g_{m}\right\}_{m \in \mathcal{I}}\right),
$$

where

(a) $\mathcal{I} \subseteq\{1, \ldots, N\}$

(b) $\left\{f_{m}\right\}_{m \in \mathcal{I}^{c}}$ and $\left\{g_{m}\right\}_{m \in \mathcal{I}^{c}}$ are generalized biorthogonal sequences

(c) $\left\{f_{m}\right\}_{m \in \mathcal{I}} \subset\left(\operatorname{span}\left\{g_{m}\right\}_{m \in \mathcal{I}^{c}}\right)^{\perp}$ and $\left\{g_{m}\right\}_{m \in \mathcal{I}} \subset\left(\operatorname{span}\left\{f_{m}\right\}_{m \in \mathcal{I}^{c}}\right)^{\perp}$ and $\left\{f_{m}\right\}_{m \in \mathcal{I}}$ and $\left\{g_{m}\right\}_{m \in \mathcal{I}}$ are A-generalized dual frames, where

$$
A=\frac{\sum_{m \in \mathcal{I}} \alpha_{m}}{\operatorname{dim}\left(\operatorname{span}\left\{f_{m}\right\}_{m \in \mathcal{I}}\right)} .
$$

Proof. Let $\left(\left\{f_{m}\right\}_{m=1}^{N},\left\{g_{m}\right\}_{m=1}^{N}\right)$ be a local extrema of the real and the imaginary part of the mixed frame potential $\widetilde{F P}: \tilde{S}\left(\left\{\alpha_{m}\right\}_{m=1}^{N}\right) \longrightarrow K$.

(1) We have that in particular $\left(\left\{f_{m}\right\}_{m=1}^{N},\left\{g_{m}\right\}_{m=1}^{N}\right)$ is a critical pair of sequences, so by Theorem 1 for each $m \in\{1, \ldots, N\}, f_{m}$ is an eigenvector of 
$T U^{\star}$ and $g_{m}$ is an eigenvector of $U T^{\star}$, and the corresponding eigenvalues are conjugates.

(2) Let $\left\{\lambda_{j}\right\}_{j=1}^{J}$ the sequence of distinct eigenvalues of $T U^{*}$, where $\lambda_{J}$ is an eigenvalue of $T U^{*}$ which satisfies that $\left|\lambda_{J}\right| \leq\left|\lambda_{j}\right|$, for all $j<J$.

Take $\left\{I_{j}\right\}_{j=1}^{J}$ the sequence of indexing sets given by

$$
I_{j}=\left\{m \in\{1, \ldots, N\}: T U^{\star} f_{m}=\lambda_{j} f_{m} \text { and } U T^{\star} g_{m}=\overline{\lambda_{j}} g_{m}\right\} \text {. }
$$

By Theorem $1\left\{f_{m}\right\}_{m \in I_{j}}$ and $\left\{g_{m}\right\}_{m \in I_{j}}$ are $\lambda_{j}$-generalized dual frames for all $j=1, \ldots, J$.

(3) We will show that $\left\{f_{m}\right\}_{m \in I_{j}}$ is linearly independent in $R_{j}$ for any $j<J$. The proof that $\left\{g_{m}\right\}_{m \in I_{j}}$ is linearly independent in $L_{j}$ for any $j<J$ is analogous.

Assume that $\left\{f_{m}\right\}_{m \in I_{j}}$ is not 1.i. in $R_{j}$ for some $j=1, \ldots, J-1$. Then there exists a nonzero sequence of $\left\{r_{m}\right\}_{m \in I_{j}} \subset K$ such that $\left|r_{m}\right| \leq \frac{1}{2}$ for all $m \in I_{j}$ and $\sum_{m \in I_{j}} \overline{r_{m}} \alpha_{m} f_{m}=0$.

We will assume without loss of generality that $\left(\left\{f_{m}\right\}_{m=1}^{N},\left\{g_{m}\right\}_{m=1}^{N}\right)$ minimizes the real part of the mixed frame potential. The other cases can be proved in a similar way.

a) If $\operatorname{Re}\left(\lambda_{J}\right)<0$ we take $h_{1} \in R_{J}$ and $h_{2} \in L_{J}$, such that $\left\langle h_{1}, h_{2}\right\rangle=1$.

Let $m \in 1, \ldots, N$ and $u_{m} \in K$ such that $u_{m}^{2}=\alpha_{m}$. We define for each $m \in 1, \ldots, N \Psi_{m}:(-1,1) \rightarrow S\left(\alpha_{m}\right), \Psi_{m}(t)=\left(\beta_{m}(t), \gamma_{m}(t)\right)$ where

$\beta_{m}(t)= \begin{cases}\sqrt{1-\operatorname{sgn}\left(\operatorname{Re}\left(\alpha_{m} \lambda_{j}\right)\right) t^{2}\left|r_{m}\right|^{2}} f_{m}+t r_{m} u_{m} h_{1}, & m \in I_{j} ; \\ f_{m}, & m \notin I_{j}\end{cases}$

and

$\gamma_{m}(t)= \begin{cases}\sqrt{1-\operatorname{sgn}\left(\operatorname{Re}\left(\alpha_{m} \lambda_{j}\right)\right) t^{2}\left|r_{m}\right|^{2}} g_{m}+t r_{m} u_{m} h_{2}, & m \in I_{j} ; \\ g_{m}, & m \notin I_{j} .\end{cases}$

We have that $\left\{\Psi_{m}(0)\right\}_{m=1}^{N}=\left(\left\{f_{m}\right\}_{m=1}^{N},\left\{g_{m}\right\}_{m=1}^{N}\right)$ and

$\operatorname{Re}(\widetilde{F P})\left(\left\{\Psi_{m}(t)\right\}_{m=1}^{N}\right)=\operatorname{Re} \sum_{m=1}^{N} \sum_{n=1}^{N}\left\langle\beta_{m}(t), \gamma_{n}(t)\right\rangle\left\langle\beta_{n}(t), \gamma_{m}(t)\right\rangle$.

By the product rule

$$
\begin{aligned}
\frac{d R e(\widetilde{F P})}{d t}\left(\left\{\Psi_{m}(0)\right\}_{m=1}^{N}\right) & =\operatorname{Re} \sum_{m \in I_{j}} \sum_{n=1}^{N}\left\langle r_{m} u_{m} h_{1}, g_{n}\right\rangle\left\langle f_{n}, g_{m}\right\rangle+ \\
& +\operatorname{Re} \sum_{m \in I_{j}} \sum_{n=1}^{N}\left\langle f_{m}, r_{n} u_{n} h_{2}\right\rangle\left\langle g_{m}, f_{n}\right\rangle+ \\
& +\operatorname{Re} \sum_{m \in I_{j}} \sum_{n=1}^{N}\left\langle f_{m}, g_{n}\right\rangle\left\langle f_{n}, r_{m} u_{m} h_{2}\right\rangle+ \\
& +\operatorname{Re} \sum_{m \in I_{j}} \sum_{n=1}^{N}\left\langle f_{m}, g_{n}\right\rangle\left\langle r_{n} u_{n} h_{1}, g_{m}\right\rangle= \\
& S_{1}+S_{2}+S_{3}+S_{4} .
\end{aligned}
$$


$S_{1}=0$ since for $m \in I_{j}$, we have that $\left\langle f_{n}, g_{m}\right\rangle=0$ for $n \notin I_{j}$ and $\left\langle h_{1}, g_{n}\right\rangle=0$ for $n \in I_{j}$ because $h_{1} \in R_{J}$. In a similar way we see that $S_{2}, S_{3}$ and $S_{4}=0$. Hence we obtain

$$
\begin{aligned}
& \frac{d R e(\widetilde{F P})}{d t}\left(\left\{\Psi_{m}(0)\right\}_{m=1}^{N}\right)=0 . \\
& \frac{d^{2} \operatorname{Re}(\widetilde{F P})}{d t^{2}}\left(\left\{\Psi_{m}(0)\right\}_{m=1}^{N}\right)=\operatorname{Re} \sum_{m \in I_{j}} \sum_{n=1}^{N}\left\langle\beta_{m}^{\prime \prime}(0), \gamma_{n}(0)\right\rangle\left\langle\beta_{n}(0), \gamma_{m}(0)\right\rangle+ \\
& +\operatorname{Re} \sum_{m \in I_{j}} \sum_{n=1}^{N}\left\langle\beta_{m}^{\prime}(0), \gamma_{n}^{\prime}(0)\right\rangle\left\langle\beta_{n}(0), \gamma_{m}(0)\right\rangle+\operatorname{Re} \sum_{m \in I_{j}} \sum_{n=1}^{N}\left\langle\beta_{m}^{\prime}(0), \gamma_{n}(0)\right\rangle\left\langle\beta_{n}(0), \gamma_{m}^{\prime}(0)\right\rangle+ \\
& +\operatorname{Re} \sum_{m \in I_{j}} \sum_{n=1}^{N}\left\langle\beta_{m}^{\prime}(0), \gamma_{n}(0)\right\rangle\left\langle\beta_{n}^{\prime}(0), \gamma_{m}(0)\right\rangle+\operatorname{Re} \sum_{m \in I_{j}} \sum_{n=1}^{N}\left\langle\beta_{m}^{\prime}(0), \gamma_{n}^{\prime}(0)\right\rangle\left\langle\beta_{n}(0), \gamma_{m}(0)\right\rangle+ \\
& +\operatorname{Re} \sum_{m \in I_{j}} \sum_{n=1}^{N}\left\langle\beta_{m}(0), \gamma_{n}^{\prime \prime}(0)\right\rangle\left\langle\beta_{n}(0), \gamma_{m}(0)\right\rangle+\operatorname{Re} \sum_{m \in I_{j}} \sum_{n=1}^{N}\left\langle\beta_{m}(0), \gamma_{n}^{\prime}(0)\right\rangle\left\langle\beta_{n}(0), \gamma_{m}^{\prime}(0)\right\rangle+ \\
& +\operatorname{Re} \sum_{m \in I_{j}} \sum_{n=1}^{N}\left\langle\beta_{m}(0), \gamma_{n}^{\prime}(0)\right\rangle\left\langle\beta_{n}^{\prime}(0), \gamma_{m}(0)\right\rangle+R e \sum_{m \in I_{j}} \sum_{n=1}^{N}\left\langle\beta_{m}^{\prime}(0), \gamma_{n}(0)\right\rangle\left\langle\beta_{n}(0), \gamma_{m}^{\prime}(0)\right\rangle+ \\
& +\operatorname{Re} \sum_{m \in I_{j}} \sum_{n=1}^{N}\left\langle\beta_{m}(0), \gamma_{n}^{\prime}(0)\right\rangle\left\langle\beta_{n}(0), \gamma_{m}^{\prime}(0)\right\rangle+R e \sum_{m \in I_{j}} \sum_{n=1}^{N}\left\langle\beta_{m}(0), \gamma_{n}(0)\right\rangle\left\langle\beta_{n}(0), \gamma_{m}^{\prime \prime}(0)\right\rangle+ \\
& +\operatorname{Re} \sum_{m \in I_{j}} \sum_{n=1}^{N}\left\langle\beta_{m}(0), \gamma_{n}(0)\right\rangle\left\langle\beta_{n}^{\prime}(0), \gamma_{m}^{\prime}(0)\right\rangle+R e \sum_{m \in I_{j}} \sum_{n=1}^{N}\left\langle\beta_{m}^{\prime}(0), \gamma_{n}(0)\right\rangle\left\langle\beta_{n}^{\prime}(0), \gamma_{m}(0)\right\rangle+ \\
& +\operatorname{Re} \sum_{m \in I_{j}} \sum_{n=1}^{N}\left\langle\beta_{m}(0), \gamma_{n}^{\prime}(0)\right\rangle\left\langle\beta_{n}^{\prime}(0), \gamma_{m}(0)\right\rangle+R e \sum_{m \in I_{j}} \sum_{n=1}^{N}\left\langle\beta_{m}(0), \gamma_{n}(0)\right\rangle\left\langle\beta_{n}^{\prime}(0), \gamma_{m}^{\prime}(0)\right\rangle+ \\
& +
\end{aligned}
$$

We obtain that $S_{2}=S_{4}=S_{5}=S_{7}=S_{10}=S_{12}=S_{13}=S_{15}=0$ and $S_{1}=S_{6}=S_{11}=S_{16}=-\sum_{m \in I_{j}}\left|r_{m}\right|^{2}\left|\operatorname{Re}\left(\alpha_{m}\right) \lambda_{j}\right|$. For the rest of the sums $S_{3}=S_{8}=S_{9}=S_{14}=\operatorname{Re}\left(\lambda_{J}\right) \sum_{m \in I_{j}}\left|r_{m}\right|^{2}\left|\alpha_{m}\right|$. Finally

$$
\frac{d^{2} \operatorname{Re}(\widetilde{F P})}{d t^{2}}\left(\left\{\Psi_{m}(0)\right\}_{m=1}^{N}\right)=4\left(-\sum_{m \in I_{j}}\left|r_{m}\right|^{2}\left|\operatorname{Re}\left(\alpha_{m} \lambda_{j}\right)\right|+\operatorname{Re}\left(\lambda_{J}\right) \sum_{m \in I_{j}}\left|r_{m}\right|^{2}\left|\alpha_{m}\right|\right),
$$

thus $\frac{d^{2} R e(\widetilde{F P})}{d t^{2}}\left(\left\{\Psi_{m}(0)\right\}_{m=1}^{N}\right)<0$, since the sequence $\left\{r_{m}\right\}_{m=1}^{N}$ is nonzero by assumption.

So in $t=0$ there is a maximum of $\operatorname{Re}(\widetilde{F P})$ restricted to $\left\{\Psi_{m}(t)\right\}_{m=1}^{N}$, i.e. we have that for all $t \in(-1,1)$

$$
\operatorname{Re}(\widetilde{F P})\left(\left\{\Psi_{m}(t)\right\}_{m=1}^{N}\right)<\operatorname{Re}(\widetilde{F P})\left(\left\{\Psi_{m}(0)\right\}_{m=1}^{N}\right)=\operatorname{Re}(\widetilde{F P})\left(\left(\left\{f_{m}\right\}_{m=1}^{N},\left\{g_{m}\right\}_{m=1}^{N}\right)\right)
$$


which is a contradiction since we assumed that $\left(\left\{f_{m}\right\}_{m=1}^{N},\left\{g_{m}\right\}_{m=1}^{N}\right)$ is a local minimizer of $\operatorname{Re}(\widetilde{F P})$.

b) If $\operatorname{Re}\left(\lambda_{J}\right) \geq 0$ we use the same function $\Psi_{m}(t)=\left(\beta_{m}(t), \gamma_{m}(t)\right)$ as defined in a), but choose $h_{1} \in R_{J}, h_{2} \in L_{J}$ such that $\left\langle h_{1}, h_{2}\right\rangle=-1$. Analogously as in a) we obtain

$$
\frac{d R e(\widetilde{F P})}{d t}\left(\left\{\Psi_{m}(0)\right\}_{m=1}^{N}\right)=0 .
$$

For the second derivative we have

$$
\frac{d^{2} \operatorname{Re}(\widetilde{F P})}{d t^{2}}\left(\left\{\Psi_{m}(0)\right\}_{m=1}^{N}\right)=4\left(\sum_{m \in I_{j}}-\left|r_{m}\right|^{2}\left|\operatorname{Re}\left(\alpha_{m} \lambda_{j}\right)\right|-\operatorname{Re}\left(\lambda_{J}\right) \sum_{m \in I_{j}}\left|r_{m}\right|^{2}\left|\alpha_{m}\right|\right) \text {. }
$$

If $\operatorname{Re}\left(\lambda_{J}\right)>0$, we know that $\frac{d^{2} R e(\widetilde{F P})}{d t^{2}}\left(\left\{\Psi_{m}(0)\right\}_{m=1}^{N}\right)<0$.

So in $t=0$ there is also a maximum of $\operatorname{Re}(\widetilde{F P})$ restricted to $\left\{\Psi_{m}(t)\right\}_{m=1}^{N}$, which is a again a contradiction since $\left(\left\{f_{m}\right\}_{m=1}^{N},\left\{g_{m}\right\}_{m=1}^{N}\right)$ is a local minimizer of $\operatorname{Re}(\widetilde{F P})$.

Now consider $\operatorname{Re}\left(\lambda_{J}\right)=0$. Let $m_{0} \in I_{j}$ such that $r_{m_{0}} \neq 0$. If $\operatorname{Re}\left(\alpha_{m_{0}} \lambda_{j}\right) \neq$ 0 , then $\frac{d^{2} R e(\widetilde{F P})}{d t^{2}}\left(\left\{\Psi_{m}(0)\right\}_{m=1}^{N}\right)<0$ and we are done.

If $\operatorname{Re}\left(\alpha_{m_{0}} \lambda_{j}\right)=0$, we are in the only case where we use the hypothesis that we also have a local extrema in the imaginary part of the restricted mixed frame potential.

Observe that if $j<J$ then $\lambda_{j} \neq 0$, since $\left|\lambda_{j}\right| \geq\left|\lambda_{J}\right|$ and $\lambda_{j} \neq \lambda_{J}$. Also, we have that $\alpha_{m_{0}} \neq 0$. So $\alpha_{m_{0}} \lambda_{j} \neq 0$, thus we know that if $\operatorname{Re}\left(\alpha_{m_{0}} \lambda_{j}\right)=0$, necessarily $\operatorname{Im}\left(\alpha_{m_{0}}\right) \lambda_{j} \neq 0$. Using the same curve $\left\{\Psi_{m}(t)\right\}_{m=1}^{N}$, but replacing in the definition $\operatorname{Re}\left(\alpha_{m} \lambda_{j}\right)$ by $\operatorname{Im}\left(\alpha_{m} \lambda_{j}\right)$ in case there is a minimum in the imaginary part, and by $-\operatorname{Im}\left(\alpha_{m} \lambda_{j}\right)$ in case there is a maximum, we also arrive to a contradiction for this particular case.

Hence we can conclude that $\left\{f_{m}\right\}_{m \in I_{j}}$ is linearly independent in $R_{j}$.

(4) As we observed before, if $j<J$ then $\lambda_{j} \neq 0$. Let $w_{j}$ be such that $w_{j}^{2}=\lambda_{j}$. We will prove that $\left\{\frac{1}{w_{j}} f_{n}\right\}_{n \in I_{j}}$ and $\left\{\frac{1}{\bar{w}_{j}} g_{n}\right\}_{n \in I_{j}}$ are biorthogonal sequences for $j<J$ :

By item (3) we have that $\left\{\frac{1}{w_{j}} f_{n}\right\}_{n \in I_{j}}$ is l.i. in $R_{j}$ and $\left\{\frac{1}{\bar{w}_{j}} g_{n}\right\}_{n \in I_{j}}$ is l.i in $L_{j}$. In item (2) we showed that for all $f \in R_{j}$,

$$
\sum_{m \in I_{j}}\left\langle f, g_{m}\right\rangle f_{m}=\lambda_{j} f
$$

so

$$
\sum_{m \in I_{j}}\left\langle f, \frac{g_{m}}{\overline{w_{j}}}\right\rangle \frac{f_{m}}{w_{j}}=f
$$

We also proved that for $f \in L_{j}$,

$$
\sum_{m \in I_{j}}\left\langle f, f_{m}\right\rangle g_{m}=\lambda_{j} f
$$


so $\left\{\frac{1}{w_{j}} f_{n}\right\}_{n \in I_{j}}$ and $\left\{\frac{1}{\overline{w_{j}}} g_{n}\right\}_{n \in I_{j}}$ are a basis of $R_{j}$ and $L_{j}$ respectively. Hence for $l \in I_{j}$ we have $f_{l} \in R_{j}$ and $g_{l} \in L_{j}$ and

$$
\begin{aligned}
0 & =\sum_{m \in I_{j}}\left\langle f_{l}, \frac{g_{m}}{\overline{w_{j}}}\right\rangle \frac{f_{m}}{w_{j}}-f_{l} \\
& =\left(\left\langle\frac{f_{l}}{w_{j}}, \frac{g_{l}}{\overline{w_{j}}}\right\rangle-1\right) f_{l}+\sum_{m \in I_{j}, m \neq l}\left\langle\frac{f_{l}}{w_{j}}, \frac{g_{m}}{\overline{w_{j}}}\right\rangle f_{m} .
\end{aligned}
$$

Since $\left\{\frac{1}{w_{j}} f_{n}\right\}_{n \in I_{j}}$ is a basis in $R_{j}$ it follows that $\left\langle\frac{f_{l}}{w_{j}}, \frac{g_{m}}{w_{j}}\right\rangle=0$ for any $l \in I_{j}$, $l \neq m, m$, and $\left\langle\frac{f_{l}}{w_{j}}, \frac{g_{l}}{w_{j}}\right\rangle=1$ and so we obtain the result.

Observe that in particular we saw that if $m \in I_{j}, j<J$ we have that $\alpha_{m}=\lambda_{j}$.

(5) By item (2) we have that for all $f \in L_{J}$

$$
\sum_{j \in I_{J}}\left\langle f, f_{j}\right\rangle g_{j}=\lambda_{J} f
$$

Let $\left\{e_{n}\right\}_{n=1}^{\operatorname{dim} L_{J}}$ be an orthonormal basis in $L_{J}$. Then

$$
\begin{aligned}
\frac{1}{\operatorname{dim} L_{J}} \sum_{m \in I_{J}} \alpha_{m} & =\frac{1}{\operatorname{dim} L_{J}} \sum_{m \in I_{J}}\left\langle f_{m}, g_{m}\right\rangle \\
& =\frac{1}{\operatorname{dim} L_{J}} \sum_{m \in I_{J}}\left\langle f_{m}, \sum_{j=1}^{\operatorname{dim} L_{J}}\left\langle g_{m}, e_{j}\right\rangle e_{j}\right\rangle \\
& =\frac{1}{\operatorname{dim} L_{J}} \sum_{m \in I_{J}} \sum_{j=1}^{\operatorname{dim} L_{J}}\left\langle f_{m}, e_{j}\right\rangle\left\langle g_{m}, e_{j}\right\rangle \\
& =\sum_{j=1}^{\operatorname{dim} L_{J}} \frac{1}{\operatorname{dim} L_{J}}\left\langle\sum_{m \in I_{J}}\left\langle f_{m}, e_{j}\right\rangle g_{m}, e_{j}\right\rangle \\
& =\sum_{j=1}^{\operatorname{dim} L_{J}} \frac{1}{\operatorname{dim} L_{J}} \lambda_{J}\left\langle e_{j}, e_{j}\right\rangle=\lambda_{J} .
\end{aligned}
$$

Similarly, we obtain

$$
\frac{1}{\operatorname{dim} R_{J}} \sum_{m \in I_{J}} \alpha_{m}=\lambda_{J}
$$

Finally, we obtain the decomposition

$$
\left\{f_{m}\right\}_{m=1}^{N}=\left\{f_{m}\right\}_{m \in I_{J} c} \cup\left\{f_{m}\right\}_{m \in I_{J}}^{N}
$$

and

$$
\left\{g_{m}\right\}_{m=1}^{N}=\left\{g_{m}\right\}_{m \in I_{J c}} \cup\left\{g_{m}\right\}_{m \in I_{J}} .
$$

By item (4) we have that $\left\{f_{m}\right\}_{m \in I_{J} c}$ and $\left\{g_{m}\right\}_{m \in I_{J c}}$ are generalized biorthogonal sequences. From item (2) and (5) it follows that $\left\{f_{m}\right\}_{m \in I_{J}}$ and $\left\{g_{m}\right\}_{m \in I_{J}}$ are $\lambda_{J}$-generalized dual frames where $\lambda_{J}=\frac{1}{\operatorname{dim} L_{J}} \sum_{m \in I_{J}} \alpha_{m}$. So, setting $I=I_{J}$, we have the desired result. 
As mentioned before, under some additional hypothesis we can assure the same structure for a pair that is a local extrema of the real or the imaginary part of the restricted frame potential:

Proposition 4. Let $\left\{\alpha_{n}\right\}_{n=1}^{N} \subset K_{\neq 0}$ and $\left(\left\{f_{m}\right\}_{m=1}^{N},\left\{g_{m}\right\}_{m=1}^{N}\right) \in \tilde{S}\left(\left\{\alpha_{m}\right\}_{m=1}^{N}\right)$ such that $T U^{*}$ is injective.

(1) If there exists an eigenvalue $\lambda_{J}$ of $T U^{*}$ such that $\operatorname{Re}\left(\lambda_{J}\right) \neq 0$, the decomposition of Theorem $\mathbf{Q}$ can be obtained assuming only that $\left(\left\{f_{m}\right\}_{m=1}^{N},\left\{g_{m}\right\}_{m=1}^{N}\right)$ is a local extrema of the real part of $\widetilde{F P}: \tilde{S}\left(\left\{\alpha_{m}\right\}_{m=1}^{N}\right) \longrightarrow K$.

(2) If there exists an eigenvalue $\lambda_{J}$ of $T U^{*}$ such that $\operatorname{Im}\left(\lambda_{J}\right) \neq 0$, the decomposition of Theorem 圆 can be obtained assuming only that $\left(\left\{f_{m}\right\}_{m=1}^{N},\left\{g_{m}\right\}_{m=1}^{N}\right)$ is a local extrema of the imaginary part of $\widetilde{F P}: \tilde{S}\left(\left\{\alpha_{m}\right\}_{m=1}^{N}\right) \longrightarrow K$.

Proof. In each case the proof is the same as the proof of Theorem 2, except that we set

$$
I=I_{J}=\left\{m: T U^{\star} f_{m}=\lambda_{J} f_{m} \text { and } U T^{\star} g_{m}=\overline{\lambda_{J}} g_{m}\right\}
$$

associated to $\lambda_{J}$ (which now not necessarily satisfies $\left|\lambda_{J}\right| \leq\left|\lambda_{j}\right|$ for all $j<J$ ). The result follows from the observations in item (3) of the proof of Theorem 2

We finally obtain the following result concerning dual frames with prescribed scalar porducts.

Corollary 1. Let $\left\{\alpha_{m}\right\}_{m=1}^{N} \subset K$. Then the following statements are equivalent:

(1) There exists $\left(\left\{f_{m}\right\}_{m=1}^{N},\left\{g_{m}\right\}_{m=1}^{N}\right) \in \tilde{S}\left(\left\{\alpha_{m}\right\}_{m=1}^{N}\right)$ which is a pair of dual frames.

(2) There exists $\left(\left\{f_{m}\right\}_{m=1}^{N},\left\{g_{m}\right\}_{m=1}^{N}\right)$ in $\tilde{S}\left(\left\{\alpha_{m}\right\}_{m=1}^{N}\right)$ such that $T U^{*}$ has only real eigenvalues, $\overline{F P}\left(\left\{f_{m}\right\}_{m=1}^{N},\left\{g_{m}\right\}_{m=1}^{N}\right)=d$ and $\operatorname{Re}\left(\sum_{m=1}^{N} \alpha_{m}\right) \geq d$.

Proof. (1) $\Rightarrow(2)$

Assume $\left(\left\{f_{m}\right\}_{m=1}^{N},\left\{g_{m}\right\}_{m=1}^{N}\right) \in \tilde{S}\left(\left\{\alpha_{m}\right\}_{m=1}^{N}\right)$ is a pair of dual frames. Then $T U^{*}=$ Id and so 1 is the only eigenvalue of $T U^{*}$, which implies that $\widetilde{F P}\left(\left\{f_{m}\right\}_{m=1}^{N},\left\{g_{m}\right\}_{m=1}^{N}\right)=$ $d$. By Proposition 2 we have $\sum_{m=1}^{N} \alpha_{m}=d$, hence in particular $\operatorname{Re}\left(\sum_{m=1}^{N} \alpha_{m}\right) \geq d$.

$(2) \Rightarrow(1)$

Take $\left(\left\{f_{m}\right\}_{m=1}^{N},\left\{g_{m}\right\}_{m=1}^{N}\right) \in \tilde{S}\left(\left\{\alpha_{m}\right\}_{m=1}^{N}\right)$ such that $T U^{*}$ has only real eigenvalues, $\widetilde{F P}\left(\left\{f_{m}\right\}_{m=1}^{N},\left\{g_{m}\right\}_{m=1}^{N}\right)=d$ and $\operatorname{Re}\left(\sum_{m=1}^{N} \alpha_{m}\right) \geq d$.

Since all the eigenvalues of $T U^{*}$ are real, by Proposition 1 we have that

$\widetilde{F P}\left(\left\{f_{m}\right\}_{m=1}^{N},\left\{g_{m}\right\}_{m=1}^{N}\right)$ and $\sum_{m=1}^{N} \alpha_{m}$ are real and $\widetilde{F P}\left(\left\{f_{m}\right\}_{m=1}^{N},\left\{g_{m}\right\}_{m=1}^{N}\right)=$ $d \geq \frac{1}{d}\left(\sum_{m=1}^{N} \alpha_{m}\right)^{2}$. Since $\sum_{m=1}^{N} \alpha_{m}=\operatorname{Re}\left(\sum_{m=1}^{N} \alpha_{m}\right) \geq d$, we obtain $d=$ 
$\frac{1}{d}\left(\sum_{m=1}^{N} \alpha_{m}\right)^{2}$, and so $\left(\left\{f_{m}\right\}_{m=1}^{N},\left\{g_{m}\right\}_{m=1}^{N}\right)$ attains the lower bound of the restricted frame potential. Hence, as we could see in the proof of Proposition 1 TU* has only one eigenvalue equal to $\frac{1}{d} \sum_{m=1}^{N} \alpha_{m}=1$

On the other hand, $\left(\left\{f_{m}\right\}_{m=1}^{N},\left\{g_{m}\right\}_{m=1}^{N}\right)$ is then also a local minima. So, by item (5) of the proof of Theorem 2, $\frac{1}{d} \sum_{m=1}^{N} \alpha_{m}=\frac{1}{\operatorname{dimL_{J}}} \sum_{m=1}^{N} \alpha_{m}$, which says that $\operatorname{dim} L_{J}=d$, i.e. $\left(\left\{f_{m}\right\}_{m=1}^{N},\left\{g_{m}\right\}_{m=1}^{N}\right)$ is a dual frame.

Remark 3. If we assume $N>d$, the statements in the previous corollary are also equivalent to say that $\sum_{m=1}^{N} \alpha_{m}=d$. This is a consequence of Proposition 2 and of Corollary 3.7 in $[$.

\section{ACKNOWLEDGMENT}

The authors want to thank Ole Christensen for useful discussions concerning this paper.

S. Heineken acknowledges the support of the Intra-European Marie Curie Fellowship (FP7 project PIEF-GA-2008-221090), UBACyT 2011-2014 (UBA) and PICT 20110436 (ANPCyT).

The research of I. Carrizo was supported by the EUCETIFA project of the University of Vienna, CONICET, Universidad Nacional de San Luis and the Technical University of Denmark.

\section{REFERENCES}

[1] J. Benedetto and D. Colella. Wavelet analysis of spectogram seizure chips. Proc. SPIE Conf. on Wavelet Appl. in Signal and Image Proc., 512-521, San Diego, CA, July 1995.

[2] J. Benedetto and M. Fickus. Finite Normalized Tight Frames. Adv. Comput. Math., 18:357385, 2003.

[3] P. Casazza. Custom Building Finite Frames. Contemp. Math., Amer. Math. Soc., Providence, 345:61-86, 2004.

[4] P. Casazza, M. Fickus, J. Kovacević, M. Leon, and J. Tremain. A Physical Interpretation of Tight Frames. Appl. Numer. Harmon. Anal., Birkhäuser Boston, Boston, MA, 2006.

[5] P. Casazza and M. Fickus. Minimizing fusion frame potential. Acta. Appl. Math., 107(103):724, 2009.

[6] O. Christensen. An Introduction to Frames and Riesz Basis. Birkhäuser Boston, Boston, MA, 2003.

[7] O. Christensen and Y. Eldar. Generalized Shift-Invariant Systems and Frames for Subspaces. J. Fourier Anal. Appl., 11(3):299-311, 2005.

[8] O. Christensen, A.M. Powell, and X.C. Xiao. A note on finite dual frame pairs Proc. Amer. Math. Soc., 140:3921-3930, 2012.

[9] I.Daubechies. The wavelet transform, time-frequency localization and signal analysis. IEEE Trans. Inform. Th., 36(5):961-1005, 1990.

[10] I.Daubechies. Ten Lectures on Wavelets. SIAM, Philadelphia, PA, 1992.

[11] R. J. Duffin and A. C. Schaeffer. A class of nonharmonic Fourier series. Trans. Amer. Math. Soc., 72:341-366, 1952.

[12] V. Goyal, J. Kovacević and J. Kelner. Quantized frame expansions with erasures. Appl. Comput. Harmon. Anal., 10: 203-233, 2001.

[13] P. Massey, M. Ruiz and D. Stojanoff. The structure of minimizers of the frame potential on fusion frames. J. Fourier Anal. Appl. 16 (4):514543, 2010. 
[14] C. Heil and D. Walnut. Continuous and discrete wavelet transforms. SIAM Rev., 31:628-666, 1989.

[15] T. Strohmer and R. Heath. Jr. Grassmanian frames with applications to coding and communications. Appl. Comput. Harmon. Anal., vol. 14 (3):257-275, 2003.

[16] S. Waldron. Generalized Welch Bound Equality sequences are tight frames. IEEE Trans. Info. Th., vol.49(9): 2307-2309, 2003. 